\title{
Evaluation of Cervical Smears by Conventional and Liquid Based Cytology
}

\author{
Prakhar Srivastava* and Rashmi Arora \\ Vardhman Mahavir Medical College and Safdarjung Hospital, West Ansari Nagar, New Delhi, India
}

\section{ABSTRACT}

Background: Cervical cancer is the second most common cancer. Screening for cervical cancer is done by Pap [Papanicolaou] test by Conventional Pap Smear [CPS] or by newer technique Liquid Based Cytology [LBC]. The aim of the study was to learn and compare cervical lesions on both the techniques.

Methods: This was a prospective study conducted over a 2 months period. Cervical smears were taken from 625 patients. On $25 / 625$ patients both CPS and LBC were done. CPS and LBC were done on 300/625 patients each. The smears were processed and stained with Papanicolaou stain. Smears were studied for adequacy, cell types, inflammatory/ haemorrhagic background, organisms and reactive changes. The cervical lesions were categorised according to the Bethesda System of classification. The two techniques were compared based on certain defined parameters.

Results: The techniques of CPS and LBC were learnt and cervical cells were appreciated on smears. Various epithelial abnormalities could be identified. Inflammation, bacterial vaginosis, candidiasis and reactive changes were noted. Out of 25 cases taken for comparison, 24 [96\%] and 25 [100\%] cases were satisfactory on CPS and LBC respectively.CPS showed more cases of severe inflammation and hemorrhagic background as compared to LBC.

Conclusions: We conclude that both CPS and LBC are effective screening modalities for cervical lesions. Despite better performance of $\mathrm{LBC}$ in many aspects, it was felt that CPS is an equally effective tool in our settings due to the cost constraints.

Keywords: Cytodiagnosis, Pap Test, Cervical Cancer, Mass Screening

\section{Introduction}

Cancer of the cervix is the most common cancer cause of death in the developing countries. ${ }^{[1]}$ Every year in India, 122,844 women are diagnosed with cervical cancer and 67,477 die from the disease. ${ }^{[2]}$ There are several methods to screen cervical cancer including Pap [Papanicolaou] test by Conventional Pap Smear [CPS] or by Liquid Based Cytology [LBC]. The screening coverage in Asian countries is low and varies from 50 per cent in Singapore to 2.6-5 per cent in India. ${ }^{[3,4,5]}$ Though data from 20 populations based cancer registries in India indicate a steady decline in cervical cancer incidence rates over the last two decades, it still occupies number two position and the risk of disease is still high. ${ }^{[6]}$

The clinical performance of CPS is not without limitations. A broad range of sensitivity [30\%-87\%] has been reported for the detection of high-grade lesions by the conventional Pap test. ${ }^{[7]}$ The conventional Pap test was also found to have a false-negative rate of about $14 \%$ to $33 \%$, approximately two-thirds of which is due to limitations of sampling or slide preparation. ${ }^{[8]}$ To overcome these limitations, Liquid Based Cytology was introduced. It is a new technique in India. The shift from CPS to LBC is because LBC provides better sample quality, reproducibility, sensitivity, and specificity, as well as the ability to perform molecular testing. ${ }^{[9]}$ The Bethesda system [TBS] is a system used for reporting Pap smear results. ${ }^{[10]}$ Latest guidelines followed are of 2014, which are an update of the 2001 Bethesda System terminology, refinements of morphologic criteria, and incorporation of revisions and additional new information into a third edition of the Bethesda atlas for cervical cytology. ${ }^{[11]}$

The following study was carried out to get a greater insight into the procedure and the analysis of cervical smears by both CPS and LBC and their applications in a developing country like India.

The aims of the study were: 1 . To learn the techniques of LBC and CPS., 2. To identify the cells in LBC and CPS., 3. To categorise the cervical lesions according to the Bethesda system.

\section{Material and Methods}

The study was conducted in the Department of Pathology and the Department of Obstetrics \& Gynaecology of 
Vardhman Mahavir Medical College \& Safdarjung Hospital, New Delhi. The study was a prospective study conducted for 2 months in which smears from 625 patients were studied.

Study Population: Patients visiting the OPD of the Department of Obstetrics \& Gynaecology, Safdarjung Hospital.

Inclusion Criteria: 1. Routine gynaecological examination of females above 25 years of age coming to the OPD. \& 2. Patients with complaints of discharge or bleeding per vagina.

Exclusion Criteria: 1. Smears obscured by blood or mucus. \& 2. Very thick smears.

\section{Methods}

After obtaining the permission and clearance from the Institute Ethics Committee of our institution, 625 patients who attended the gynaecology out-patient department during a period of 2 months from June 2016 to July 2016 were randomly selected. On 300 patients, CPS was performed. On another 300 patients, LBC was performed. On the remaining 25 patients, both CPS and LBC were performed for comparison. The chief complaints of these patients were bleeding per vaginum, white discharge, irregular menstrual cycles, lower-abdominal pain etc.

CPS was made using an Ayer's spatula and an endocervical brush, which was immediately fixed using the cyto preservative. LBC sample was collected using the detachable brush provided by the manufacturer. The plastic brush was rinsed in vial of LBC medium containing cytorich preservative and was kept for minimum 40 minutes.

LBC Smears were prepared using BD Prep Stain TM Slide Processor [SurePath Technique] [12] according to the directions in the operators' manual provided by the manufacturer.

CPS were stained by Pap staining method. ${ }^{[13]}$ The slides were studied for the cell morphology. The cervical lesions were categorised according to the Bethesda System of classification. ${ }^{[1]}$ Only satisfactory smears were included for statistical analysis.

\section{Results}

CPS covered the entire slide while LBC smears had a monolayer of cells spread over $13 \mathrm{~mm}$ diameter only [Fig. $1 \mathrm{AC}]$. LBC smear showed lesser debris, cell clumps and obscuring elements microscopically. The background was cleaner in LBC as compared to CPS [Fig. 1B, 2D]. Immediate liquid fixation in $\mathrm{LBC}$ prevented artefacts such as air-drying.
All the smears were studied in detail for the following parameters:

1. Adequacy

2. Cell types

3. Inflammatory background

4. Haemorrhagic background

5. Organisms

6. Impression

7. Reactive changes

The superficial cells were seen as large polygonal cells, with eosinophilic, transparent cytoplasm and small dark nuclei. Intermediate squamous cells were similar in size to superficial cells or somewhat smaller. They had a vesicular nucleus with usually a basophilic cytoplasm [Fig. 2]. Parabasal cells occurred singly and were usually round or oval in shape, with smooth cytoplasmic borders and bland and homogenous nuclei [Fig. 3]. Basal cells had scant basophilic cytoplasm and large nuclei with chromatin granules and tiny round nucleoli. Endocervical cells were seen as columnar cells, arranged in palisades or in honeycomb pattern .

CPS: Out of 300 cases of CPS, 29 [9.7\%] were unsatisfactory. For the 29 unsatisfactory cases, the main causes were scant cellularity of squamous cells in 15 [51.7\%] cases and haemorrhage in 10 [34.5\%] cases. The diagnosis was made as Negative for intraepithelial lesion or malignancy [NILM] in 265 [97.8\%] of cases. Atypical glandular cells of unknown significance [AGUS] were reported in $2[0.7 \%]$ cases. Atypical squamous cells of unknown significance [ASCUS] were reported in 2 [0.7\%] cases. High-grade squamous intra epithelial lesion [HSIL] was reported in $2[0.7 \%]$ cases which showed nuclear changes like increased N/C ratio and irregular coarsely clumped chromatin.

The background was seen for the presence of inflammation or haemorrhage. The background was clear in 38 [14\%] cases. The severity of inflammation was graded as $1+$, $2+$ and $3+$ based on the visual impression of the extent of neutrophils present. Inflammation was $1+$ in 126 [46.5\%] cases, $2+$ in 98 [36.1\%] cases and $3+$ in 29 [10.8\%] cases. Haemorrhage was seen exclusive of inflammation in 20 $[7.4 \%]$ cases.

The background was analysed for the type of organisms present. Mixed flora was found in $6[2.2 \%]$ cases, fungal Candida hyphae were reported in $2[0.7 \%]$ cases and shift in flora was reported in 34 [12.5\%] cases. The reactive changes such as atrophy and therapy associated changes were appreciated in 8 [2.9\%] cases. 
LBC: Out of 300 cases of LBC, 14 [4.7\%] were unsatisfactory, the main cause being scant cellularity of squamous cells in 11 [78.6\%] out of 14 unsatisfactory cases. The diagnosis was made as Negative for intraepithelial lesion or malignancy [NILM] in 282 [98.6\%] of cases. Atypical squamous cells of unknown significance [ASCUS] were reported in $2[0.7 \%]$ cases. Low-grade squamous intra-epithelial lesion [LSIL] [Fig. 4] was reported in 1 $[0.3 \%]$ case which showed the presence of koilocytes. Squamous cell carcinoma [Fig. 5] was reported in 1 [0.3\%] case which showed abnormal cells, necrosis and mitosis.

The background was clear in 39 [13.6\%] cases. The background showed inflammation as $1+$ in 110 [38.4\%] cases, $2+$ in 109 [38.1\%] cases and $3+$ in 38 [13.3\%] cases. Haemorrhagic background was seen in 4 [1.4\%] cases.
Mixed flora was seen in 4 [1.4\%] cases, Shift in flora was reported in 31 [10.8\%] cases and budding yeast and Candida hyphae were seen in $1[0.3 \%]$ case. The reactive changes such as atrophy and therapy associated changes were appreciated in $9[3 \%]$ cases.

Out of 25 cases taken for comparison, 24 [96\%] cases of CPS were satisfactory while 25 [100\%] of LBC cases were satisfactory. Other details are given in Table: 1.

\section{Discussion}

The importance of Pap smears in the screening of cervical cancer was reported in the studies by Manjit et al. ${ }^{[14]}$ and Mulazim et al. ${ }^{[15]}$ Out of $9.7 \%$ unsatisfactory cases of CPS, the main causes were scant cellularity of squamous cells in 15 [51.7\%] cases and haemorrhage in 10 [34.5\%] cases.

Table 1: Comparison between CPS and LBC [N=25].

\begin{tabular}{|c|c|c|c|c|}
\hline S. No. & CRITERIA & CATEGORISATIONS & CPS [N=25] & LBC $[\mathrm{N}=25]$ \\
\hline \multirow{2}{*}{1.} & \multirow{2}{*}{ Adequacy } & Satisfactory & $24[96 \%]$ & $25[100 \%]$ \\
\hline & & Unsatisfactory & $1[4 \%]$ & 0 \\
\hline \multirow{2}{*}{2.} & \multirow[t]{2}{*}{ Impression } & NILM & $24[100 \%]$ & $25[100 \%]$ \\
\hline & & Bacterial vaginosis & $1[4 \%]$ & 0 \\
\hline \multirow{3}{*}{3.} & \multirow{3}{*}{$\begin{array}{l}\text { Inflammatory } \\
\text { background }\end{array}$} & $1+$ & $11[46 \%]$ & 19 [76\%] \\
\hline & & $2+$ & $6[25 \%]$ & $5[20 \%]$ \\
\hline & & $3+$ & 7 [29\%] & $1[4 \%]$ \\
\hline 4. & Haemorrhage & & $1[4 \%]$ & 0 \\
\hline 5. & EC cells & & $17[71 \%]$ & $21[84 \%]$ \\
\hline
\end{tabular}

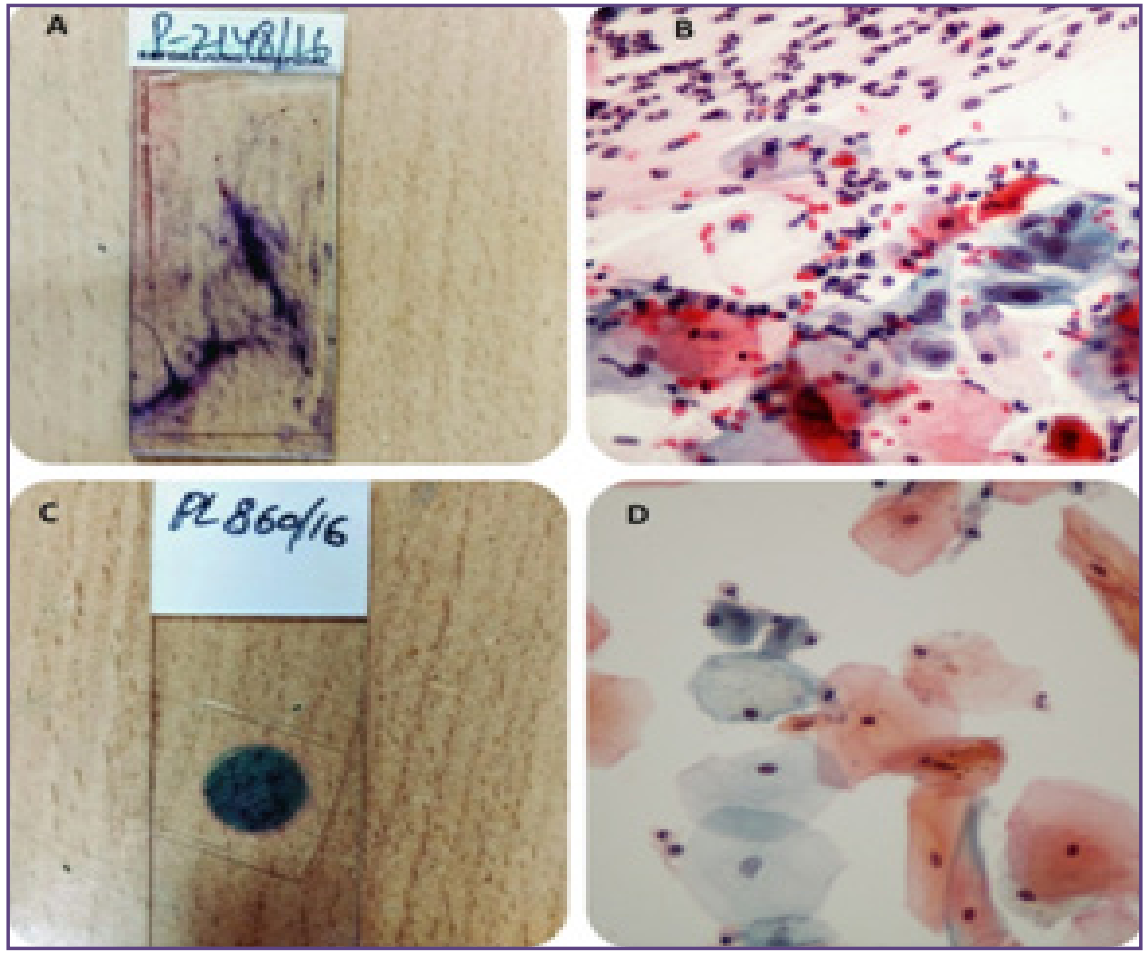

Fig. 1: Comparison between CPS and LBC. [A] CPS Slide. [B] A negative CPS [pap, x400]. [C] LBC Slide. [D] A negative LBC Smear [pap, x400]. 


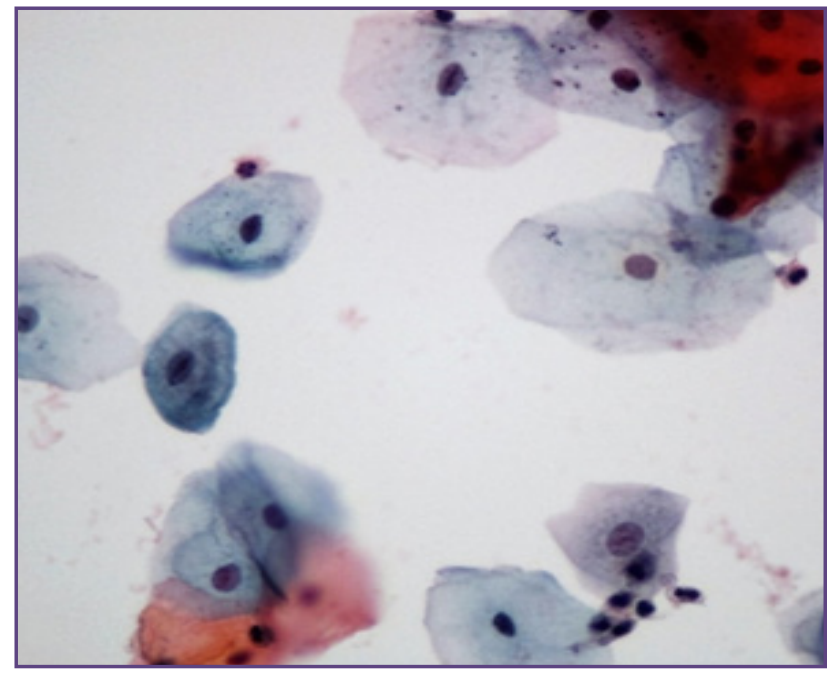

Fig. 2: An LBC smear showing intermediate squamous cells [Pap, $\mathrm{x}$ 400].

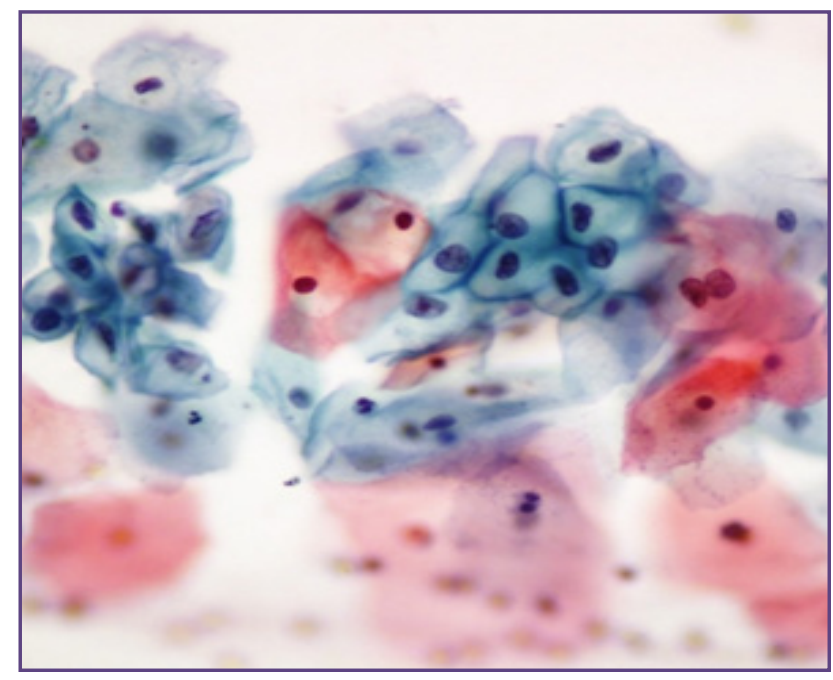

Fig. 4: An LBC smear showing LSIL [Pap, x400].

The Jyotsna et al. ${ }^{[16]}$ study also noted haemorrhage, dense inflammation, drying artefacts and scant cellularity in the unsatisfactory cases of CPS. Similarly, the Gupta N et al. [17] study reported that the main cause of unsatisfactory rate in CPS was low cellularity followed by low cellularity with excess blood. The interpretation was made as NILM, AGUS, ASCUS and HSIL. No case of LSIL or carcinoma was reported probably because of the smaller sample size and limited duration of the study.

In CPS, inflammation was $1+$ in $46.5 \%$ cases and $3+$ in $10.8 \%$ cases. Haemorrhage was seen in $7.4 \%$ cases. Since the smears are directly prepared from the brush, and stained and visualised as such, the background is inflammatory and hemorrhagic in so many cases. The Singh VB et al.

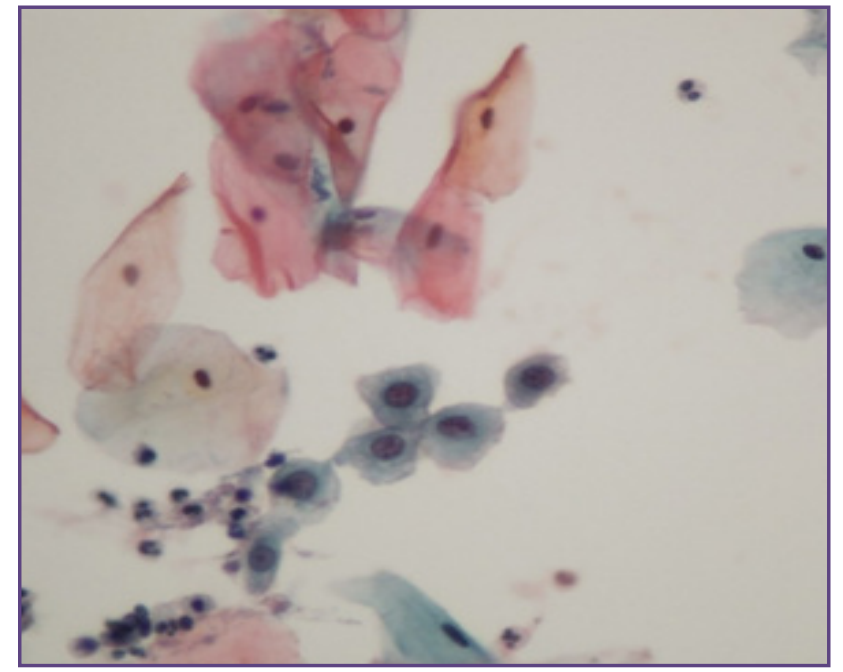

Fig. 3: An LBC smear showing parabasal cells [Pap, $x 400]$.

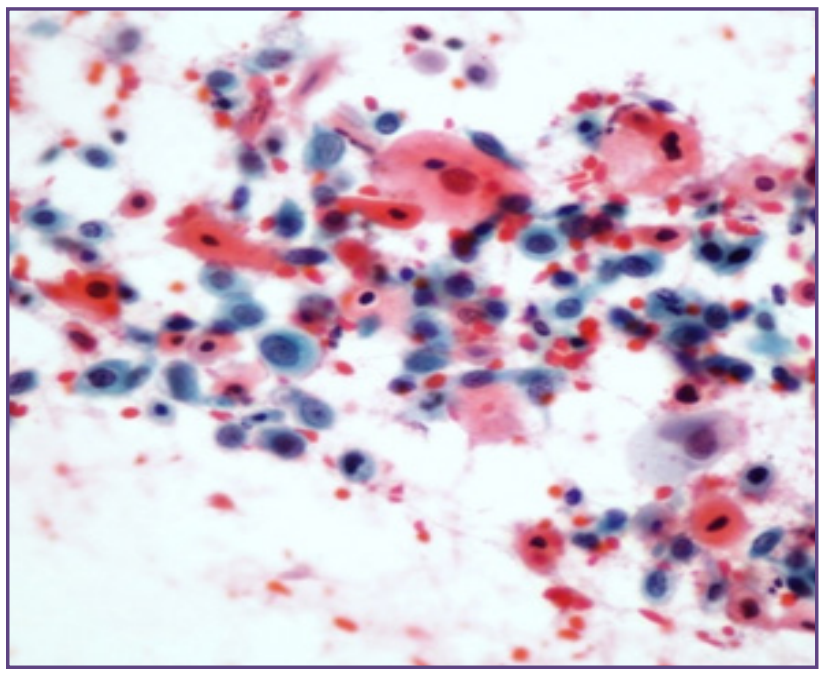

Fig. 5: An LBC smear showing squamous cell carcinoma of the cervix [Pap, $x 400]$.

${ }^{[18]}$ study also found increased cases of haemorrhage and inflammatory samples in CPS.

Out of $4.7 \%$ unsatisfactory cases of LBC, the main cause was scant cellularity of squamous cells in $78.6 \%$ of unsatisfactory cases. The studies done by Siebers et al. ${ }^{[19]}$ and Gupta $\mathrm{N}$ et al. ${ }^{[17]}$ found scant cellularity as the sole cause for unsatisfactory LBC, similar to our study.

In LBC, the background was clear in 39 [13.6\%] cases. $1+$ inflammation was found in 110 [38.4\%] cases and 3+ in 38 [13.3\%] cases. Haemorrhagic background was seen in $1.4 \%$ cases. The background was clearer and showed lesser inflammation due to the removal of the obscuring materials like blood and mucus during the processing of the sample. Despite this, inflammation was usually not 
missed out in LBC because as reported by other authors, neutrophils are clearly visualised on LBC although their number is reduced. ${ }^{[16,20]}$

Mixed flora, shift in flora, budding yeast cells and Candida hyphae were reported were reported with both CPS and LBC. While the test may detect infections, this is not its primary purpose. ${ }^{[1]}$ The reactive changes such as atrophy and therapy associated changes were also appreciated.

Out of 25 cases taken for comparison, 24 [96\%] cases of CPS were satisfactory while 25 [100\%] of LBC cases were satisfactory. Most of the other studies reported higher satisfactory rates with LBC as compared to CPS. [16, 18, $19,21,22,23]$ The diagnosis was NILM in $24[100 \%]$ of the satisfactory CPS cases and in $25[100 \%]$ of the satisfactory LBC cases. No difference in interpretation of Pap lesion was reported in our comparative study. However, in studies done by Abulafia et al. [24], Obwegeser et al. ${ }^{[25]}$, Vincenzo et al. ${ }^{[26]}$, Davey et al. ${ }^{[27]}$, an interpretation of ASCUS was more frequent with CPS, while no significant difference was reported in LSIL/HSIL detection. Our study group was small and so probably larger study group is required for a definite opinion.

Bacterial vaginosis was reported in 1 [4\%] case of LBC while none of the CPS smear showed any shift in flora. Similarly, in the Sherwani et al. ${ }^{[28]}$ study, where only infectious organisms were taken into account, CPS detected organisms in $3.1 \%$ smears while LBC detected them in $8.7 \%$ cases.

CPS showed more cases of severe inflammation whereas inflammation was appreciated as mild in most of the LBC smears. In CPS, the background showed inflammation as $1+$ in 11 [45.8\%] cases, $2+$ in 6 [25\%] cases and 3+ in $7[29 \%]$ cases. In LBC, the background was $1+$ in 19 [76\%] cases, $2+$ in 5 [20\%] cases and 3+ in 1 [4\%] case. Similarly, the study by Jyotsna et al. ${ }^{[16]}$, reported $3+$ inflammation more in cases of CPS [42\%] than in LBC [20\%] while $1+$ was more in LBC [52\%] than in CPS [31\%]. Haemorrhagic background and RBCs were encountered more frequently in CPS [1 [4\%]] whereas none of the LBC smear showed haemorrhage.

More number of endocervical cells was reported in LBC, which is in accordance to the studies by Bergerone et al. [29] and Sharma J et al. ${ }^{[16]}$ However, the study by Strander et al. ${ }^{[30]}$ found that most LBC smears lacked endocervical cells as compared to CPS.

\section{Conclusion}

Both the techniques of CPS and LBC were performed and learnt in detail on 625 patients. Different cell types were identified and their staining and cytomorphological features were appreciated. Different cervical lesions were seen and their characters were analysed and diagnosed according to the Bethesda classification.

Some advantages of LBC that were identified are higher satisfactory rate, better spread of cells, cleaner background, smaller screening area and easy to review over CPS. Despite these advantages, it is felt that CPS remains as an equally effective tool for cervical screening in developing countries like India due to the significantly higher costs of LBC.

\section{Acknowledgement}

Dr Indrani Dhawan, Head of the Department, Department of Pathology, Vardhman Mahavir Medical College and Safdarjung Hospital, New Delhi

\section{References}

1. World Cancer Report, World Health Organization. 2014. pp. Chapter 5.12. ISBN 9283204298.

2. ICO Information Centre on HPV and cancer. Human Papillomavirus and Related Diseases in India, 2014.

3. Nor HO, Matejka R. Challenges to cervical cancer screening in a developing country: The case of Malaysia. Asia Pacific J Cancer Prev. 2009;10:747-52.

4. World Health Survey. Geneva: WHO; 2003. WHO.

5. Gakidou E, Nordhagen S, Obermeyer Z. Coverage of cervical cancer screening in 57 countries: low average levels and large inequalities. PloS Med. 2008;5:e132

6. Three year report of population based cancer registries 2006-2008. New Delhi: ICMR; 2010. National Cancer Registry Programme.

7. American College of Obstetricians and Gynecologists. ACOG Practice Bulletin No. 99: management of abnormal cervical cytology and histology. Obstet Gynecol 2008; 112:1419-1444.

8. Hartmann K, Hall SA, Nanda K, et al. Systematic Evidence Review Number 25: Screening for Cervical Cancer. Rockville, MD: US Department of Health and Human Services; [Accessed June 10, 2016].

9. Gibb RK, Martens MG. The Impact of Liquid-Based Cytology in Decreasing the Incidence of Cervical Cancer. Reviews in Obstetrics and Gynecology 2011;4[Suppl 1]:S2-S11.

10. The 1988 Bethesda System for reporting cervical/vaginal cytological diagnoses. National Cancer Institute Workshop. JAMA. 1989 Aug 18;262[7]:931-4.

11. Wilbur DC, Nayar R. Bethesda 2014: improving on a paradigm shift. Cytopathology 2015 Dec;26[6]:339-42.

12. BD PrepStain Slide Processor Operator's Manual; Becton, Dickinson and Company; 2007.

13. Koss LG, Melamed MR. Koss Diagnostic Cytology and its Histopathologic Bases. Fifth ed: Lippincott Williams and Wilkins Publications; 2005. 
14. Bal MS, Goyal R, Suri AK, Mohi MK. Detection of abnormal cervical cytology in Papanicolaou smears. Journal of Cytology / Indian Academy of Cytologists 2012;29[1]:45-47.

15. Bukhari MH, Saba K, Qamar S, Majeed MM, Niazi S, Naeem S. Clinicopathological importance of Papanicolaou smears for the diagnosis of premalignant and malignant lesions of the cervix. Journal of Cytology / Indian Academy of Cytologists 2012;29[1]:20-25.

16. Sharma J, Toi PCh, Siddaraju N, Sundareshan M, Habeebullah S. A comparative analysis of conventional and SurePath liquid-based cervicovaginal cytology: A study of 140 cases. J Cytol 2016;33[2]:80-4.

17. Gupta N, Bhar VS, Rajwanshi A, Suri V. Unsatisfactory rate in liquid-based cervical samples as compared to conventional smears: A study from tertiary care hospital. Cytojournal 2016 Jun 10;13:14.

18. Singh VB, Gupta N, Nijhawan R, Srinivasan R, Suri V, Rajwanshi A. Liquid-based cytology versus conventional cytology for evaluation of cervical Pap smears: experience from the first 1000 split samples. Indian J Pathol Microbiol 2015 Jan-Mar;58[1]:17-21.

19. Siebers AG, Klinkhamer PJ, Vedder JE, Arbyn M, Bulten J. Causes and relevance of unsatisfactory and satisfactory but limited smears of liquid-based compared with conventional cervical cytology. Arch Pathol Lab Med 2012 Jan;136[1]:76-83.

20. Roghaei MA, Afshar Moghaddam N, Pooladkhan SH, Roghaie SH. Adequacy criteria and cytomorphological changes in Liquid-Prep TM versus conventional cervical cytology. SEJM 2010;11:173-82.

21. Sigurdsson K. Is a liquid-based cytology more sensitive than a conventional Pap smear? Cytopathology 2013 Aug;24[4]:254-63.

22. Ronco G, Confortini M, Maccallini V, Naldoni C, Segnan N, Sideri M, Zappa M,Zorzi M, Calvia M, Giorgi Rossi
P. [Health technology assessment report. Use of liquidbased cytology for cervical cancer precursors screening]. Epidemiol Prev 2012 Sep-Oct;36[5 Suppl 2]:e1-e33.

23. Budak MŞ, Senturk MB, Kaya C, Akgol S, Bademkiran MH, Tahaoğlu AE, Yildirim A, Büyükbayram H. A comparative study of conventional and liquid-based cervical cytology. Ginekol Pol 2016;87[3]:190-3.

24. Abulafia O, Pezzullo JC, Sherer DM. Performance of ThinPrep liquid-based cervical cytology in comparison with conventionally prepared papanicolaou smears: A quantitative survey. Gynecol Oncol 2003:137-44.

25. Obwegeser JH, Brack S. Does liquid-based technology really improve detection of cervical neoplasia? A prospective, randomized trial comparing the ThinPrep Pap Test with the conventional Pap Test, including follow-up of HSIL cases. Acta Cytol 2001 Sep-Oct;45[5]:709-14.

26. Ilter E, Midi A, Haliloglu B, Celik A, Yener A, Ulu I, et al. Comparison of conventional and liquid based cytology: Do the diagnosis benefits outweigh the financial aspect? Turk J Med SCI. 2012;42:1200-6.

27. Davey E, Barratt A, Irwig L, Chan SF, Macaskill P, Mannes $\mathrm{P}$, et al. Effect of study design and quality on unsatisfactory rates, cytology classifications, and accuracy in liquidbased versus conventional cervical cytology: A systemic review. Lancet 2006;367:122-32.

28. Sherwani RK, Khan T, Akhtar K, Zeba A, Siddiqui FA, Rahman K, et al. A comparative study of conventional pap smears and liquid based cytology. J Cytol 2007;24:167-72.

29. Bergeron C, Fagnani F. Performance of a new, liquid-based cervical screening technique in the clinical setting of a large French laboratory. Acta Cytol 2003;47:753-61.

30. Strander B, Andersson-Ellström A, Milsom I, Rådberg T, Ryd W. Liquid-based cytology versus conventional Papanicolaou smear in an organized screening program: A prospective randomized study.Cancer 2007;111:285-91.

*Corresponding author:

Prakhar Srivastava, D-44, Dhanwantri Block, New Boys Hostel, Safdarjung Hospital, West Kidwai Nagar, New Delhi, India- 110023

Phone: +91 07042875202

Email: sriprakhar1996@gmail.com

Financial or other Competing Interests: None.

Date of Submission : 19.04.2017

Date of Acceptance : 14.08.2017

Date of Publication : 19.12.2017 